\title{
Dynamic Compressed Sensing for Real-time Tomographic Reconstruction
}

Jonathan Schwartz ${ }^{1}$, Huihuo Zheng ${ }^{2}$, Marcus Hanwell ${ }^{3}$, Yi Jiang ${ }^{2}$ and Robert Hovden ${ }^{1}$

${ }^{1}$ University of Michigan, Ann Arbor, Michigan, United States, ${ }^{2}$ Argonne National Laboratory, Lemont, Illinois, United States, ${ }^{3}$ Kitware, Clifton Park, New York, United States

Electron tomography reveals three-dimensional (3D) structure of biological specimens [1,2] and inorganic materials [3-5] across the nano- to meso- scale. However, 3D reconstruction suffers from an insufficient number of projections and a limited angular range inside the microscope, commonly referred to as the missing wedge [6]. To overcome these experimental limitations, advancements in tomographic algorithms utilizes compressed sensing (CS) methods that leverage the notion of sparsity to provide superior 3D resolution from limited projection angles and at lower doses [7-8]. While compressed sensing algorithms provide higher quality reconstructions, they require substantially more computational resources to complete and significantly prolong electron tomography. Even worse, incomplete convergence and poor selection of tunable parameters are often discovered at the end of an arduous reconstruction - multiplying the total time required to produce a high-quality 3D tomogram. Scientists would benefit from immediate feedback to begin interpreting 3D specimen structure and optimize experimental parameters to accelerate the end-to-end scientific process.

Here, we have developed a dynamic compressed sensing framework that offers 3D specimen reconstruction in real-time as experimental projection data is collected. The reconstruction algorithm begins immediately upon acquiring the first projection and dynamically updates the $3 \mathrm{D}$ structure as new projections arrive (Fig. 1a) - unlike traditional methods that start after the experiment is complete. Dynamic CS is a continuously running optimization that accommodates additional constraints provided when new projections arrive or allows on the fly modification of reconstruction parameters. Using scanning transmission electron microscope (STEM) tomography, we demonstrate dynamic CS accelerates the final reconstruction convergence by a factor of 2-3 over conventional CS algorithms. High-quality reconstructions allow meaningful interpretation of 3D nanostructure midway into a tomography experiment. Figure 1 shows the internal pores, sharp facets, and $\sim 5 \mathrm{~nm}$ surface particles on $\mathrm{SrTiO}_{3}$ nanocubes are well defined at $\sim 60 \%$ of the experimental acquisition time. This means researchers can start analysis and characterization with the high-fidelity of compressed sensing before an experiment is complete. After all the data is collected, dynamic CS further reduces reconstruction error that outpaces traditional compressed sensing.

Moreover, dynamic compressed sensing can be used to manipulate the data-tolerance constraint during the reconstruction to efficiently optimize reconstruction accuracy without completely restarting the algorithm (Fig. 2). In compressed sensing, a user-defined data-tolerance parameter controls the weight between total variation (TV) minimization and data fidelity. Our algorithm is implemented under the constrained optimization framework: $\operatorname{argmin}_{\mathrm{x}}|\mathrm{x}| \mathrm{TV}$ s.t. $|\mathrm{Ax}-\mathrm{b}| 2 \leq \varepsilon$, where $\varepsilon$ is the data-tolerance that accounts for all sources of data inconsistency such as noise and system imperfections. Tuning the datatolerance is often task-dependent and incorrectly selecting the parameter can lead to insidious artifacts [9]. We demonstrate that the data tolerance, $\varepsilon$, can be tuned interactively to visualize different constraints in real-time as the reconstruction progresses. The parameter can either be reduced to produce a sharper reconstruction (Fig. 2a) or increased to generate a smoother reconstruction (Fig. 2b). When $\varepsilon$ is incrementally increased or decreased, dynamic CS converges to solutions defined by the final $\varepsilon$ chosen 
(Fig. 2c). If $\varepsilon$ is too low the reconstruction appears noisy; if $\varepsilon$ is too high, detail and resolution are degraded.

Dynamic CS demands an efficient implementation of the algorithm to ensure the computation runs faster than the experimental data acquisition rate. Here we improved the time-per-iteration by parallelizing the most expensive computation: data reconstruction (e.g. algebraic reconstruction technique) and TVminimization with OpenMP and Message Passing Interface (MPI). For single-axis tomography, the volumetric reconstruction is distributed into independent calculations on different 2D slices orthogonal to the tilt axis [10]. 3D TV-minimization decomposes the volume into smaller 3D subdomains to compute local 3D gradients and broadcast its isotropic TV norm. With complete parallelization of compressed sensing, tomograms of up to 512 voxels $^{3}$ can be reconstructed dynamically on modest multi-core laptops or desktops during an electron tomography experiment; larger volumes ( $>1024$ voxels ${ }^{3}$ ) can be achieved with high-performance computing (HPC) resources. With the use of current petascale Leadership-class supercomputers, dynamic CS can enable real-time reconstructions from large CCD cameras, accommodating fast data acquisition rates, and hyperspectral or ptychographic tomography.

Dynamic CS provides real-time analysis of 3D specimens as an experiment progresses to produce a highquality reconstruction before the completion of data collection. The accuracy and convergence of dynamic CS demonstrates compressed sensing is a relatively convex optimization process with solutions primarily determined by the data and the data-tolerance parameter. This allows compressed sensing to reconstruct dynamically during intermittent data arrival and utilize the tomographic acquisition time for highthroughput tomography-without sacrificing reconstruction quality. Many industries, such as semiconductor device failure, require near-immediate metrology which dynamic compressed sensing accommodates. Combined with real-time 3D visualization tools [11] and robust automated data acquisition, dynamic CS can enable real-time electron tomography [12]. 


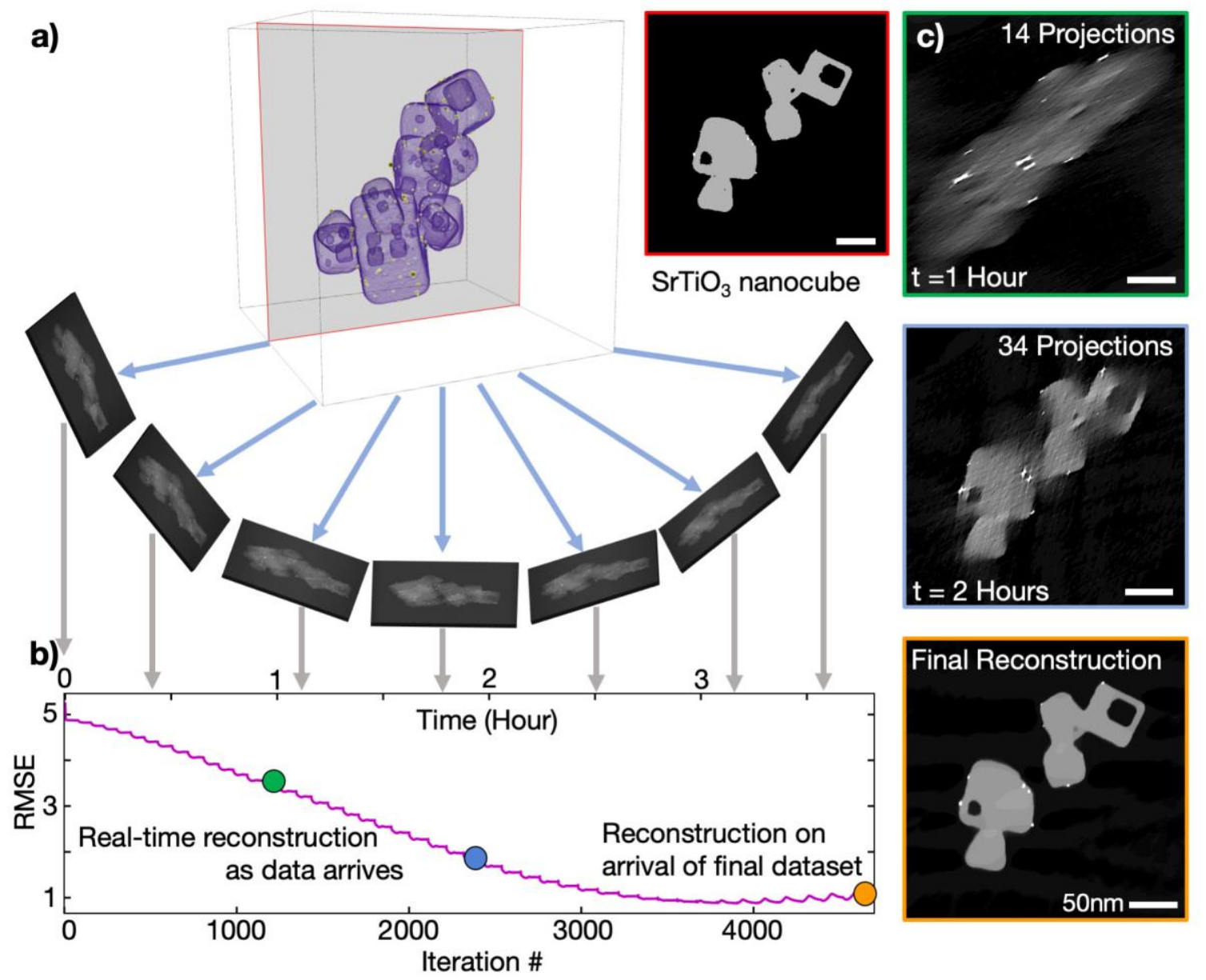

Figure 1. The dynamic CS framework demonstrated on STEM tomography of a SrTiO3 nanocube. a) As the tomographic experiment progresses, projections are collected across an angular range (typically $\pm 75^{\circ}$ for electron tomography). Measured projections are fed into the dynamic CS algorithm immediately when collected to improve the reconstruction quality. b) The root-mean-square error (RMSE) of a 3D tomogram is reduced with additional projection and optimization iterations (time). c) 2D slices of the 3D volumes shown at various time stamps $(\mathrm{t}=1,2$ (hour) and completion). The final reconstruction matches the true object illustrated in the top right of (a). 
a)
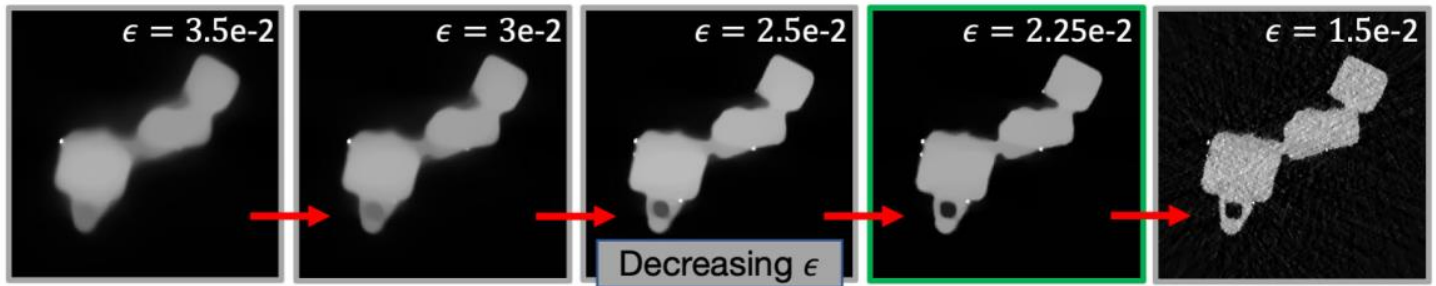

b)
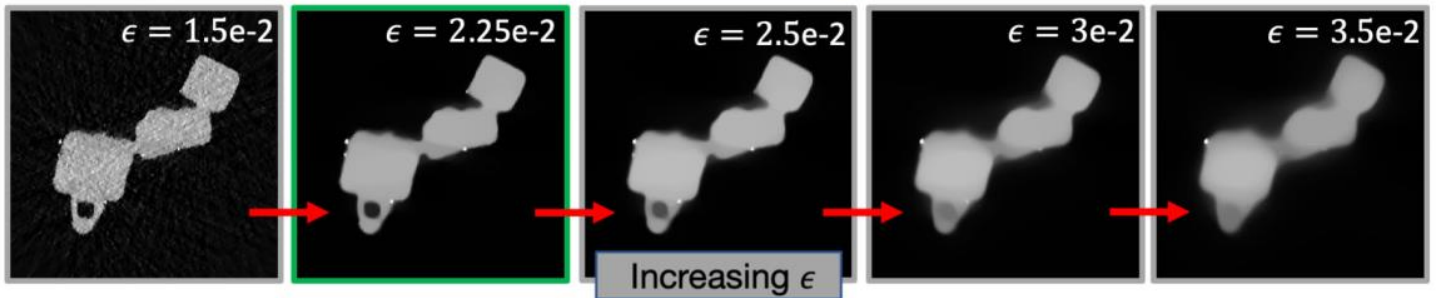

c)
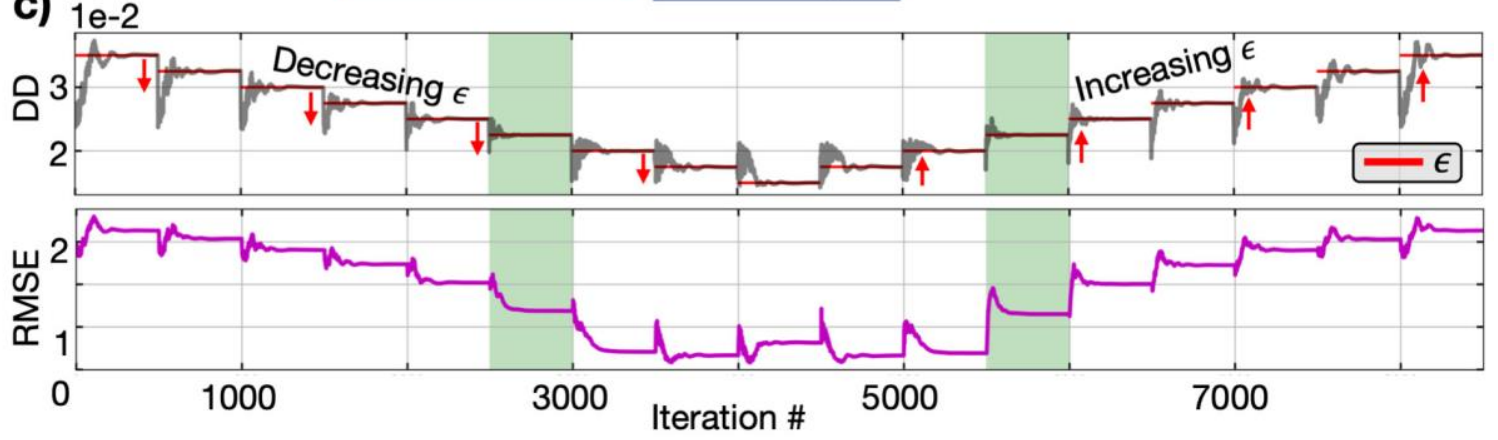

Figure 2. Dynamic manipulation of data tolerance parameter $\varepsilon$. During a dynamic CS reconstruction, scientists can manipulate $\varepsilon$ without having to reset the algorithm. The data-distance parameter $(\mathrm{DD}=\mid \mathrm{Ax}$ - b|2) measures the inconsistencies between reprojections and experimental data and controls the strength of TVmin to converge on $\varepsilon$. a-b) Shows 2D tomographic slices of a 3D SrTiO3 reconstruction when $\varepsilon$ is tightened (a) and loosened (b) mid-reconstruction. c) Plots demonstrating the progression DD and RMSE vs iterations. The area shaded in green highlights where $\varepsilon$ produces the visually desirable tomogram.

\section{References}

[1] R. David and A. Klug. Nature, 217, 130 (1968).

[2] R. Fernandez, et. al. J. Cell Biol. 188, 145 (2010).

[3] Y. Yu, et. al. Nano Letters 12, 4417 (2011).

[4] H. Sai, et. al. Science 341, 530 (2013).

[5] P. Ercius, L. Gignac, C. Hu, and D. Muller, Microsc. Micro. 15, 244 (2009).

[6] Y. Jiang, E. Padgett, R. Hovden and D. Muller, Ultramicroscopy 186, 94 (2017).

[7] D. Donoho IEEE Trans. Inf.Theor. 52, 1289 (2006).

[8] E. Candes, J. Romber, and T. Tao, IEEE Trans. Inf. Theor. 52, 489 (2006).

[9] L. Pfister and Y. Bresler. Proc. SPIE Computational Imaging 90200, 90200H11 (2014).

[10] J. Fernández, D. Gordon and R. Gordon. J. Parallel Distrib. Comput. 68, 626 (2008).

[11] R. Hovden, et. al. Microsc. Microanal. 24 (S1), 556 (2018).

[12] This research used resources of the Argonne Leadership Computing Facility, which is a DOE Office of Science User Facility supported under Contract DE-AC02-06CH11357. 